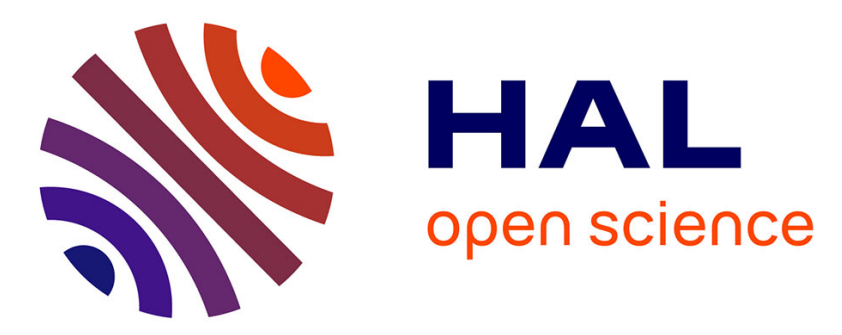

\title{
Anthropologie et droit
}

Baudouin Dupret, Irene Lizzola

\section{To cite this version:}

Baudouin Dupret, Irene Lizzola. Anthropologie et droit. Anthropen - Le dictionnaire francophone d'anthropologie ancré dans le contemporain , 2020, 10.47854/IMHA9973 . hal-03084201

\section{HAL Id: hal-03084201 https://hal.science/hal-03084201}

Submitted on 20 Dec 2020

HAL is a multi-disciplinary open access archive for the deposit and dissemination of scientific research documents, whether they are published or not. The documents may come from teaching and research institutions in France or abroad, or from public or private research centers.
L'archive ouverte pluridisciplinaire HAL, est destinée au dépôt et à la diffusion de documents scientifiques de niveau recherche, publiés ou non, émanant des établissements d'enseignement et de recherche français ou étrangers, des laboratoires publics ou privés. 


\section{Anthropologie et droit}

Dupret, Baudouin, Lizzola, Irene (2020) "Anthropologie et droit", dans Anthropen.org, Paris, Éditions des archives contemporaines., DOI:https://doi.org/10.47854/IMHA9973

\section{Link: https://www.anthropen.org/voir/Anthropologie\%20et\%20droit}

La naissance d'une discipline

Montesquieu peut sans doute être considéré comme l'un des ancêtres de l'anthropologie du droit quand il insiste sur le caractère relatif des lois de chaque peuple (Vale 2012). C'est toutefois largement en concomitance avec l'essor des empires coloniaux qu'apparaissent des «proto-anthropologues du droit» (Goodale 2017: 9), à la fois administrateurs coloniaux et académiciens, qui s'engagent dans l'étude d'un droit conçu comme révélateur de l'évolution des sociétés, de leurs cultures et des «civilisations» (voir Maine 1861; Morgan 1877). Leur conception du droit et de son rapport à la société est évolutionniste. Elle les conduit à considérer le rôle du droit dans le passage des sociétés «primitives» ou «sauvages», où le droit aurait été absent, au stade «civilisé», où le droit servirait à réguler les relations sociales en les contractualisant (Goodale 2017: 11).

La tendance culturaliste a longtemps imprégné l'anthropologie du droit. Elle fut le propre non seulement des écoles historique, évolutionniste et comparative, mais aussi de l'anthropologie coloniale, culturelle et interprétative (Dupret 2006b: 14ss). Le prisme culturaliste a également inspiré le courant du pluralisme juridique (voir infra). Essentialiste et évolutionniste, le culturalisme a amené les tenants de la discipline à considérer le droit comme l'expression particulière d'un peuple et de sa spécificité.

L'interprétativisme anthropologique se situe dans le sillage de l'anthropologie culturelle. Clifford Geertz (1983) en est le représentant majeur. Son disciple, Lawrence Rosen (1989, 2000), dans son étude sur le tribunal du qadi de la petite ville de Séfrou, dans le Moyen-Atlas marocain, considère que la justice y est caractérisée par une tendance à la négociation en continu des intérêts individuels, ce qui se reflète dans le domaine juridique. Rosen cherche à montrer, d'une part, que la décision de justice est le produit de considérations culturelles au travers desquelles le qadi mesure l'incidence de son jugement et de l'acte incriminé sur le tissu relationnel de l'univers social en question. D'autre part, il considère aussi que le cas particulier de Séfrou est une incarnation de la culture marocaine, elle-même une incarnation de la culture musulmane. 
Des auteurs comme Bronislaw Malinowski (1922, 1926) incarnent une tendance fonctionnaliste, qui affirme l'idée du droit comme expression des structures sociales et des normes qui les régissent. Avec Malinowski et Isaac Shapera (1938), l'étude du droit des sociétés «primitives» s'élargit et se combine à celle des normes sociales en vigueur, tout en se distanciant d'une vision qui ne reconnaîtrait que le droit de dérivation étatique. Ne faisant toutefois pas la distinction entre droit et ordre social, les catégories employées par ces anthropologues pour décrire les ordres juridiques en question demeurent ethno- et étatocentriques.

La question des catégories descriptives utilisées par les chercheurs en sciences sociales pour décrire le droit non occidental est à l'origine de l'un des débats majeurs en anthropologie du droit. La démarche d'anthropologues comme Max Gluckman (1955), qui a employé des catégories juridiques occidentales pour détailler le raisonnement des juges Barotse de la Rhodésie du Nord, s'oppose à celle menée par d'autres qui, comme Paul Bohannan (1957), tiennent à différencier les catégories analytiques occidentales des catégories «populaires» (folk) ou «indigènes» (native) produites in situ et in vivo.

Le courant du réalisme juridique américain, pionnier dans l'étude des pratiques du droit considérées dans leurs contextes de déroulement et d'énonciation, a été caractérisé par des prises de position divergentes quant à la définition des règles et des faits, ainsi que de leurs relations réciproques. Si, d'un côté, la position des «sceptiques à l'égard des règles» considèrent ces dernières comme le produit de la seule œuvre «créatrice» des juges ou de toute autorité définissant les contours du «droit» (voir par exemple Llewellyn et Hoebel 1941), les «sceptiques à l'égard des faits» insistent sur la variabilité et la subjectivité de l'interprétation donnée à ceux-ci au niveau judiciaire (Dupret 2006b: 144).

Dans l'ouvrage The Cheyenne Way: Conflict and Case Law in Primitive Jurisprudence, Llewellyn et Hoebel (1941) analysent une série de trouble cases (situations conflictuelles) remontant au XIXe siècle, qui leur ont été relatés dans les années 1930 par des membres des tribus Cheyenne. Les auteurs cherchent à identifier et décrire ce qu'ils appellent des law ways (façons de faire du droit) ou law stuff (des questions de droit) qu'on peut discerner dans les modes de résolution des controverses propres à cette «culture» tribale (Conley et O’Barr 2006: 179-180).

Bien que leur approche soit jugée «néo-évolutionniste» par certains anthropologues (voir par exemple Mehrotra 2001), qui reprochent à l'ouvrage de reproduire la dichotomie entre «sociétés modernes» et «sociétés primitives», The Cheyenne Way est un exemple d'analyse de cas (case analysis) avant l'heure. Il faudra attendre les travaux de Gluckman et Bohannan pour 
voir ce type d'analyse se développer et contribuer à la description ethnographique des évènements.

Dans cette perspective réaliste, le droit est traité comme une forme de régulation s'imposant aux membres de la communauté qui s'y retrouvent et qui y font référence. En cas de dispute, le droit prévaut sur tout autre ordre normatif, du fait qu'il est énoncé par une autorité communautaire (Redfield 1942).

Le regard anthropologique porté sur l'objet droit se complexifie au cours des années 1970, quand le droit commence à être étudié dans son fonctionnement propre, ainsi que dans sa relation aux normes sociales et à leur production (Moore 1969). À partir de ce moment, les études anthropologiques commencent à s'intéresser aux thèmes de la médiation et de la résolution des conflits (voir par exemple Comaroff et Roberts 1981) ou aux actes de langage à travers lesquels se formulent la représentation des normes et du droit (voir par exemple Greenhouse 1982; Starr et Collier 1989). L'anthropologie du droit se mêle alors à l'analyse de discours. Inspiré par Foucault et, plus encore, par le réalisme américain, cette analyse vise à rendre compte des enjeux de domination ancrés dans le discours juridique et des relations que ce dernier entretient avec l'idéologie et le pouvoir politiques (voir par exemple Mertz 2007; Conley et O'Barr 1990; Haviland 2003). Cette perspective permet de déconstruire l'apparence de «neutralité» du droit et de son langage, le droit étant alors étudié par les anthropologues comme un outil de reproduction des inégalités sociales.

Le pluralisme juridique

Plusieurs auteurs s'inscrivent dans le courant, initié par Eugen Ehrlich (1936), du pluralisme juridique, qui remet en question la suprématie de l'État en matière de production juridique. Masaji Chiba (1986) et Bonaventura de Sousa Santos (1989) incarnent une phase postmoderne, qui élargit le pluralisme à tout le spectre normatif.

Par conséquent, il existerait une série de «droits informels» réglant les relations sociales et différents du «droit officiel». Dans la même veine, John Griffiths (1999) affirme que le pluralisme juridique prend en compte les différentes formes de pouvoir et d'autorité présentes dans la société et exerçant un contrôle social alternatif à celui de l'État. De Sousa Santos (1989) emploie pour sa part le concept d'«interlégalité» en s'éloignant du pluralisme juridique tel que conçu par l'anthropologie du droit traditionnelle.

Aussi bien Werner Menski (2006) que Samia Bano (2012) mobilisent le concept d'agency (agentivité), estimant qu'il convient de tenir compte des différentes formes d'acteurs 
demandant la reconnaissance de droits qui entrent en conflit avec le droit positif ou qui ne sont pas «satisfaits» par ce dernier.

L'apport du pluralisme juridique a permis de sortir d'une perspective universaliste homogénéisant les critères d'identification du droit. Cette approche a permis de focaliser l'attention sur l'agentivité individuelle et les possibilités d'action et de revendication qui s'ouvrent aux individus (ou aux groupes) et à la construction desquelles ils participent. Cependant, ce mouvement confère à la «culture» une place prépondérante dans le déploiement du raisonnement juridique et dans la conception des facteurs qui ont influencé la constitution des «traditions juridiques» (Glenn 2010).

Les tenants de la première vague du pluralisme juridique rapportaient le pluralisme au cadre étatique de référence (Vanderlinden 1972). Les représentants de la deuxième vague (Griffiths 1986) ont élargi le spectre du pluralisme à tout le champ social. Quant à la troisième vague de ce courant (voir par exemple Lebel-Grenier 2002), elle considère l'ensemble de la production normative des individus au cours de leurs interactions. Cette perspective présente le danger de ne pas saisir le caractère situé de la production juridique et son entremêlement avec d'autres niveaux de normativité. La notion de «pluralisme normatif» (Dupret 1999) permet de sortir de cette impasse en soulignant à la fois la spécificité du droit, distinct d'autres formes normatives, et sa sensibilité à d'autres ordres de normativité.

Dans la foulée des débats autour du pluralisme juridique, l'école oxfordienne d'anthropologie du droit a développé le concept de «juridisme» (legalism) pour décrire les «règles généralisantes» et les «catégories abstraites» mobilisées par les acteurs pour «décrire le monde et ordonner la vie communautaire et sociale», indépendamment de l'existence d'un cadre étatique de production normative (Pirie 2019: 5). Les tenants du «juridisme» s'expriment en faveur d'une anthropologie juridique capable de tracer non seulement la présence des normes dans les pratiques, mais aussi dans les énoncés et les expressions des perceptions propres aux acteurs qui y font référence verbalement.

Sociologie critique et praxéologique, nouvelles tendances

La sociologie du droit en France a longtemps été influencée par l'œuvre pionnière d'Émile Durkheim, qui distingue les «sanctions répressives», relevant de la «solidarité mécanique», et les sanctions «restitutives», propres à la logique de la «solidarité organique» (Durkheim 1967 dans Dupret 2006b: 62). En distinguant entre sanction juridique directe et sanction morale «diffuse», propre à la conscience collective, la sociologie durkheimienne a permis de réfléchir à l'évolution parallèle du droit et de la morale. À la suite de Durkheim, la sociologie du droit a 
permis d'ancrer la norme juridique dans son milieu social et politique, et de la considérer comme étant à la fois acteur et objet du politique (Commaille 2004).

Du côté anglo-saxon, la sociological jurisprudence a permis de ne plus voir le droit comme un simple outil de contrôle social. Ce courant est très hétérogène, cependant, entre des auteurs fonctionnalistes, qui considèrent le droit comme un instrument d'organisation rationnelle et efficace des conduites sociales (Pound 1959), et d'autres qui décrivent la société comme fondée sur «le pouvoir, la coercition et la contrainte» (Hills 1971).

L'étude du droit en action se situe à la suite du courant réaliste américain, des socio-legal studies en Angleterre (Travers 2002) et de l'expérience de la revue Law and Society, fondée aux États-Unis dans les années 1960. Elle porte sur un droit ancré dans un contexte social et sur les représentations que se font les acteurs du droit, ce qu'on désigne aujourd'hui sous le terme de conscience juridique (Ewick et Silbey 1998).

Du côté des approches critiques (voir par exemple les critical legal studies), le droit est avant tout pensé comme outil de domination. Bourdieu $(1986,1991)$ décrit le langage du droit comme imbriqué dans les enjeux de légitimation du pouvoir étatique et comme le monopole des juristes qui en détiennent le capital symbolique et culturel spécifique. Foucault $(1975,1976)$ et les postmodernes considèrent le droit comme un cadre dans lequel s'inscrit l'ensemble des «micro-pouvoirs disciplinaires» qui agissent parallèlement à l'appareil juridique étatique.

S'appuyant sur l'ethnographie de la pratique du droit au sein du Conseil d'État, l'anthropologue Bruno Latour (2002) analyse les «formes concrètes de véridiction» (Raymond et al. 2004: 160) propres à cette dernière. En rejetant à la fois l'approche «internaliste» (selon laquelle le droit ne doit être étudié que d'un point de vue juridique) et l'approche «externaliste» (qui ne voit le droit que comme un dérivé du social), Latour s'engage dans l'étude du droit et de ses «associations» à l'univers social. Il montre comment les décisions prises par les juges du Conseil d'État sont construites sur la base à la fois d'une série de références au droit positif et sur des «aspects extra-juridiques» définissant l'orientation des références juridiques applicables - et que le juge veut appliquer - au cas d'espèce.

Dans ces analyses du droit «tel qu'il se fait» (Latour 2002: 205) ou du «droit en action» (Dupret 2006a), on retrouve les éléments constitutifs du droit positif qui ne sont pas «juridiques» stricto sensu mais qui relèvent d'autres ordres normatifs, dont la morale.

La conception praxéologique du droit en action reproche au pluralisme juridique d'être incapable de saisir le droit positif dans sa spécificité, toutes les expressions normatives étant considérées comme du droit. À l'inverse, l'ethnographie ethnométhodologique du travail 
juridique (Cicourel 1968; Sudnow 1965; Dupret 2006a) s'est attachée à étudier l'organisation pratique du travail des acteurs, leur raisonnement pratique et leur usage des catégories juridiques, qui s'insère dans un ensemble de contraintes normatives, contingentes et situées.

Récemment, l'anthropologie du droit s'est enrichie et ouverte à l'étude de domaines auparavant inexplorés, tels que les questions liées aux revendications et à la reconnaissance des droits des communautés autochtones (Bellier 2019), l'étude du juridisme au-delà du prisme des catégories du droit positif de dérivation étatique et dans ses expressions transnationales et supranationales (Pirie 2013; Merry 2006, 1992), ou l'approche de l'objet droit en tant qu'outil du pouvoir politique et économique et source de réaffirmation des inégalités sociales et économiques (Goodale et Clarke 2010). Le droit n'est plus seulement envisagé comme l'outil permettant de satisfaire aux besoins capitalistes des grandes entreprises multinationales et des États (Goodale et Clarke 2010) et, en ce sens, incapable d'accompagner un processus de changement social (Hirschl 2004; Pottage 2004), mais aussi comme un instrument de mobilisation, de contestation et de revendication (Goodale et Merry 2007).

\section{Références}

Bano S., 2012, Muslim Women and Shari'ah Councils: Transcending the Boundaries of Community and Law. Londres, Palgrave Macmillan.

Bellier I., 2019, «La reconnaissance des peuples autochtones comme sujets du droit international. Enjeux contemporains de l'anthropologie politique en dialogue avec le droit». Clio \& Themis - Revue électronique d'histoire du droit, no15, p.1-24. http:// cliothemis.com/La-reconnaissance-des-peuples

Bohannan P., 1957, Justice and Judgment among the Tiv. Oxford, Oxford University Press.

Bourdieu P., 1986, «La force du droit: éléments pour une sociologie du champ juridique». Actes de la recherche en sciences sociales, no64, p.3-19. https://doi.org/10.3406/ arss.1986.2332), le 25 janvier 2019.

—, 1991, Language and Symbolic Power. Cambridge, Harvard University Press.

Chiba M., 1986, Asian indigenous law: in interaction with received law. New York et Londres, Routledge et Kegan Paul.

Cicourel A., 1968, The Social Organization of Juvenile Justice. New York, John Wiley \& Sons.

Comaroff J. et S. Roberts, 1981, Rules and Processes: The Cultural Logic of Dispute in an African Context. Chicago, University of Chicago Press. 
Commaille J., 2004, «Sociologie politique», dans L. Cadiet (dir.), Dictionnaire de la justice, Paris, PUF.

Conley J. et W. O’Barr, 1990, Rules versus Relationships: The Ethnography of Legal Discourse. Chicago, University of Chicago Press.

-, 2006, «A Classic in Spite of Itself: The Cheyenne Way and the Case Method in Legal Anthropology», Law and Social Inquiry, vol.29, no1, p.179-217. https://doi.org/10.1111/ j.1747-4469.2004.tb00333.x

De Sousa Santos B., 1989, «Law: A Map of Misreading - Toward a Postmodern Conception of Law». Journal of Law and Society, vol.14, no3, p.279-302. https://www.jstor.org/stable/ 1410186

Dupret B., 1999, «Legal pluralism, normative plurality and the Arab world», dans B. Dupret, M. Berger et L. Al-Zwaini, Legal Pluralism in the Arab World, Leyde, Brill, p. 29-40.

—, 2006a, Le Jugement en action. Ethnométhodologie du droit, de la morale et de la justice en Egypte. Genève et Le Caire, Librairie Droz et CEDEJ, coll. «Travaux en sciences sociales» no207. —, 2006b, Droit et Sciences Sociales. Paris, Armand-Colin.

Durkheim E., 1967, De la division du travail social. Paris, Presses universitaires de France.

Ehrlich E., 1936, Fundamental Principles of the Sociology of Law. Cambridge, Harvard University Press.

Ewick P. et S. Silbey, 1998, The Common Place of Law. Chicago, University of Chicago Press.

Foucault M., 1975, Surveiller et punir. Naissance de la prison. Paris, Gallimard.

—, 1976, Histoire de la sexualité. Paris, Gallimard.

Geertz C., 1983, Local Knowledge: Further Essays in Interpretive Anthropology. New York, Basic Books.

Glenn P., 2010, Legal traditions of the world: Sustainable diversity in law. New York, Oxford University Press.

Gluckman M., 1955, The Judicial Process among the Barotse of Northern Rhodesia. Manchester, Manchester University Press.

Goodale M., 2017, Anthropology of Law: A Critical Introduction. New-York, New-York University Press.

Goodale M. et K.M. CLARKE, 2010, Understanding the Multiplicity of Justice. In Mirrors of Justice: Law and Power in the Post-Cold War Era. New York, Cambridge University Press.

Goodale M. et S.E. Merry (dir.), 2007, The Practice of Human Rights: Tracking Law between the Global and the Local. Cambridge, Cambridge University Press.

Greenhouse C.J., 1982, «Looking at Culture, Looking for Rules». Man (New Series), vol.17, no1, p.58-73. https://www.jstor.org/stable/2802101 
Griffiths J., 1986, «What is Legal Pluralism?» TheJournal of Legal Pluralism and Unofficial Law, vol.18, no24, p.1-55. https://doi.org/10.1080/07329113.1986.10756387

—, 1999, «Preface», dans B. Dupret, M. Berger et L. Al-Zwaini, Legal Pluralism in the Arab World, Leyde, Brill, p.vii-x.

Haviland J.B., 2003, «Ideologies of Language: Some Reflections on Language and U.S. Law». American Anthropologist, no105, p.764-774. doi:10.1525/aa.2003.105.4.764

Hills S.L., 1971, Crime, Power and Morality. New York, Harper Collins.

Hirschl R., 2004, Towards Juristocracy: The Origins and Consequences of the New Constitutionalism. Cambridge, Harvard University Press.

Latour B., 2002, La fabrique du droit. Une ethnographie du Conseil d'État. Paris, La Découverte, coll. Armillaire.

Lebel-Grenier S., 2002, Pour un pluralisme juridique radical. Thèse de doctorat, faculté de droit, Université McGill.

Llewellyn K.N. et E.A. Hoebel, 1941, The Cheyenne Way: Conflict and Case Law in Primitive Jurisprudence. Norman, University of Oklahoma Press.

Mehrotra A.K., 2001, «Law and the "Other": Karl N. Llewellyn, Cultural Anthropology, and the Legacy of The Cheyenne Way». Law \& Social Inquiry, vol.26, no3, p.741-775. https:// www.jstor.org/stable/829116

Maine H.S., 1861, Ancient Law: Its Connection with the Early History of Society, and Its Relation to Modern Ideas. Londres, John Murray.

Malinowski B., 1922, Argonauts of the Western Pacific: An Account of Native Enterprise and Adventure in the Archipelagoes of Melanesian New Guinea. Londres, Routledge et Kegan Paul. —, 1926, Crime and Custom in Savage Society. Londres, Kegan Paul.

Menski W., 2006, Comparative Law in a Global Context: The Legal Systems of Asia and Africa. Cambridge, Cambridge University Press.

Merry S.E., 2006, «Transnational Human Rights and Local Activism: Mapping the Middle». American Anthropologist, no108, p.38-51. https://doi.org/10.1525/ aа.2006.108.1.38

Mertz E., 2007, The Language of Law School: Learning to «Think Like a Lawyer». New York, Oxford University Press.

Moore S.F., 1969, «Law and Anthropology». Biennial Review of Anthropology, no6, p.252-300. https://www.jstor.org/stable/2949194

Morgan L.H., 1877, Ancient Society. Londres, MacMillan \& Company.

Pirie F., 2013, The Anthropology of Law. Oxford, Oxford University Press, Clarendon Law Series. 
—, 2019, «Legalism: a turn to history in the anthropology of law». Clio \& Themis - Revue électronique d'histoire du droit, no15, p.1-15. https://www.cliothemis.com/Legalism-a-turnto-history-in-the

Pottage A., 2004, «Introduction: The Fabrication of Persons and Things», dans A. Pottage et M. Mundy (dir.), Law, Anthropology, and the Constitution of the Social, Cambridge, Cambridge University Press, p.1-39.

Pound R., 1959, Jurisprudence, tome 2. St. Paul, West Publishing Company.

Raymond B.A. de et al., 2004, «Droit, réflexivité et sciences sociales. Autour du livre de Bruno Latour: La Fabrique du droit (confrontations)». Terrains \& travaux, vol.1, no6, p.159-180. https://www.cairn.info/revue-terrains-et-travaux-2004-1-page-159.htm

Redfield R., 1942, «Compte rendu de l'ouvrage The Cheyenne Way. Conflict and Case Law in Primitive Jurisprudence, par K. Llewellyn et E.A. Hoebel», University of Chicago Law Review, vol.9, no2, article 20. https://chicagounbound.uchicago.edu/uclrev/vol9/iss2/20

Rosen L., 1989, The anthropology of justice: law as culture in Islamic society. New York, Cambridge University Press.

—, 2000, The justice of Islam. Oxford, Oxford University Press.

Schapera I., 1938, A Handbook of Tswana Law and Custom: Compiled for the Bechuanaland Protectorate Administration. Oxford, Oxford University Press, International African Institute. Starr J. et J. Collier (dir.), 1989, History and Power in the Study of Law: New Directions in Legal Anthropology. Ithaca, Cornell University Press.

Sudnow D., 1965, «Normal Crimes: Sociological Features of the Penal Code in a Public Defender Office». Social Problems, vol.12, no3, p.255-276.https://doi.org/10.2307/798932

Travers M., 2002, «Symbolic Interactionism and Law»,dans R. Banakar et M. Travers (dir.), An Introduction to Law and Social Theory, Oxford et Portland, Hart Publishing.

Vale M., 2012, «Custom, Combat, and the Comparative Study of Laws: Montesquieu revisited», dans P. Desch et H. Skoda (dir.), Legalism: Anthropology and History, Oxford, Oxford University Press, p.261-278.

Vanderlinden J., 1972, Les pluralismes juridiques. Bruxelles, Éditions de l'Université de Bruxelles. 